Relations industrielles

Industrial Relations

\title{
Articulation institutionnelle et orientations du système de formation professionnelle au Québec
}

\section{Jean Charest}

Volume 54, numéro 3, 1999

URI : https://id.erudit.org/iderudit/051249ar

DOI : https://doi.org/10.7202/051249ar

Aller au sommaire du numéro

\section{Éditeur(s)}

Département des relations industrielles de l'Université Laval

\section{ISSN}

0034-379X (imprimé)

1703-8138 (numérique)

Découvrir la revue

\section{Citer cet article}

Charest, J. (1999). Articulation institutionnelle et orientations du système de formation professionnelle au Québec. Relations industrielles / Industrial Relations, 54(3), 439-471. https://doi.org/10.7202/051249ar

\section{Résumé de l'article}

Le système québécois de formation professionnelle a connu des transformations importantes au cours des années 90. La nouvelle articulation institutionnelle de ce système a été traversée par diverses influences politiques et économiques, dont une caractéristique majeure est la participation soutenue des principaux acteurs des relations industrielles. Nous proposons ici une analyse de ce que nous nommons la modernisation du système de formation professionnelle au Québec en retraçant les orientations de départ et leur logique sous-jacente, en identifiant les influences extérieures permettant de spécifier l'expérience québécoise et en concluant sur les principaux enjeux qui confrontent les acteurs.
Tous droits réservés (C) Département des relations industrielles de l'Université Laval, 1999
Ce document est protégé par la loi sur le droit d'auteur. L'utilisation des services d'Érudit (y compris la reproduction) est assujettie à sa politique d'utilisation que vous pouvez consulter en ligne.

https://apropos.erudit.org/fr/usagers/politique-dutilisation/ 


\title{
Articulation institutionnelle et orientations du système de formation professionnelle au Québec
}

\author{
JEAN CHAREST
}

Le système québécois de formation professionnelle a connu des transformations importantes au cours des années 90. La nouvelle articulation institutionnelle de ce système a été traversée par diverses influences politiques et économiques, dont une caractéristique majeure est la participation soutenue des principaux acteurs des relations industrielles. Nous proposons ici une analyse de ce que nous nommons la modemisation du système de formation professionnelle au Québec en retraçant les orientations de départ et leur logique sousjacente, en identifiant les influences extérieures permettant de spécifier l'expérience québécoise et en concluant sur les principaux enjeux qui confrontent les acteurs.

Situer la trajectoire du système québécois de formation professionnelle par rapport à d'autres expériences ou d'autres systèmes nationaux est un exercice difficile, mais stimulant sur le plan de l'analyse comparative et de l'évaluation de la politique publique. Pour ce faire, nous proposons dans cet article de revenir d'abord sur les fondements stratégiques de la modernisation du système de formation professionnelle du Québec, ce qui nous ramène au début des années 90 . D'emblée, cela permettra de répondre en partie à la question des influences extérieures au Québec qui ont inspiré cette stratégie. Également, cela permettra d'identifier les composantes majeures de ce que nous appelons la construction d'une nouvelle articulation institutionnelle en matière de formation professionnelle. Ensuite, nous situerons la trajectoire ou la stratégie du Québec par rapport à certaines expériences étrangères, notamment en nous appuyant sur une typologie des

- CHAREST, J., École de relations industrielles, Université de Montréal, Montréal, Québec.

- Nous tenons à remercier la professeure Ginette Dussault pour ses commentaires sur une version antérieure de ce texte. L'auteur demeure évidemment entièrement responsable du présent texte. 
systèmes nationaux de formation professionnelle, sur des études de cas nationaux réalisées par divers experts et sur une évaluation globale récente effectuée par le Bureau international du Travail (BIT).

Tant notre analyse que nos conclusions doivent toutefois être balisées et ce, de deux manières. D'une part, il faut considérer que le système de formation professionnelle qui s'élabore au Québec est encore très jeune et qu'il repose sur une nouvelle articulation institutionnelle essentiellement mise en place dans les années 90 . À cet égard, nous ferons état de la complexité et du temps nécessaire pour mettre en place une nouvelle articulation institutionnelle qui concerne autant d'acteurs, de logiques, de niveaux, de cultures et de préférences. D'autre part, l'analyse comparative ne doit pas être perçue comme une recherche du modèle étranger idéal qu'il suffirait ensuite de transposer dans les économies nationales. Nous soumettons ici que face à des enjeux similaires en matière d'adaptation économique et de formation professionnelle, les réponses concrètes au sein des entreprises et des marchés du travail nationaux sont filtrées par les institutions, les pratiques historiques et les acteurs en place au niveau national ou subnational (régional ou sectoriel). Qui plus est, les objectifs mêmes et les stratégies des acteurs en regard de la formation professionnelle sont sujets à la négociation à tous les niveaux, de l'entreprise jusqu'aux politiques publiques, ce qui nous renvoie forcément à considérer les systèmes nationaux de formation professionnelle sous l'angle des rapports particuliers qui animent les acteurs dans les différents systèmes de relations industrielles.

\section{LA MODERNISATION DU SYSTẼME DE FORMATION PROFES- SIONNELLE AU QUÉBEC : LES PRINCIPAUX ÉLEMENTS}

C'est au début de la décennie actuelle que la plupart des principaux éléments de la modernisation du système québécois de formation professionnelle ont été esquissés. On les retrouve dans l'Énoncé de politique sur le développement de la main-d'œuvre Partenaires pour un Québec compétent et compétitif (MMSRFP 1991). Trois aspects méritent d'être relevés ici pour les fins de notre objet : l'analyse qu'on y faisait du rôle de la formation professionnelle dans le développement économique, le diagnostic de la situation québécoise et la stratégie mise de l'avant. Sous ces trois aspects, on peut déceler, souvent explicitement, les influences extérieures qui motivaient la démarche proposée.

\section{Une main-d'œuvre qualifiée : variable stratégique pour le développement économique}

En premier lieu, l'analyse faite dans cet Énoncé quant au rôle d'une main-d'œuvre qualifiée dans le nouveau contexte économique de 
libéralisation et de mondialisation des échanges élevait cette main-d'œuvre au premier rang des variables stratégiques pour le développement économique. D'entrée de jeu, l'Énoncé évoquait que pour demeurer dans la course à la compétitivité, il fallait compter sur "..."l'ultime ressource" du Québec, sa main-d'œuvre" (MMSRFP 1991 : 9). On y soulignait aussi avec force que le gouvernement ne pouvait pas relever à lui seul les défis de la formation ; il fallait compter sur les autres acteurs agissant en partenariat. Ce partenariat était d'ailleurs présenté comme : "...la caractéristique commune des économies à succès " (MMSRFP $1991: 8$ ).

Cet attribut stratégique que l'Énoncé accordait à la main-d'œuvre, et plus spécifiquement comme on le verra à la nécessaire qualification de cette main-d'œuvre, s'inscrivait tout à fait dans le sens d'une littérature américaine influente à l'époque. Nous pensons particulièrement ici aux travaux de Robert Reich (1992) qui occupa le poste de Secrétaire au travail lors du premier mandat de Bill Clinton, de Lester Thurow (1992) et de Michael Porter $(1990,1991)$. Chez les deux premiers, la thèse centrale était assez identique et nous la résumons sommairement ainsi : dans le nouveau contexte économique des années 90 , qui se caractérise par une concurrence accrue et par une mobilité internationale du capital plus grande, le fer de lance pour assurer la compétitivité des économies industrialisées sera le développement des compétences de la main-d'œuvre (à ce sujet, voir aussi l'analyse de Sack et al. 1993). Michael Porter, dont les services furent requis par plusieurs gouvernements dont celui du Canada (Porter 1991), appuyait cette thèse du rôle stratégique d'une main-d'œuvre de plus en plus qualifiée en l'intégrant à la conception dynamique et systémique du développement économique qu'il présentait sous la forme du " losange de l'avantage concurrentiel $»^{2}$. Dans son étude diagnostique du Canada, Porter soulignait d'ailleurs qu'un des problèmes de l'économie canadienne était le peu d'importance que les employeurs du secteur privé accordaient à la formation professionnelle permanente (1991 : 57). Enfin, rappelons que Porter insistait sur l'importance du rôle des contraintes externes à l'entreprise comme éléments de stimulation dans la gestion et l'innovation au sein de celle-ci (par

1. Cette idée est exprimée clairement dans l'énoncé suivant de Reich : «The real economic challenge facing the United States in the years ahead - the same as that facing every other nation - is to increase the potential value of what its citizens can add to the global economy, by enhancing their skills and capacities and by improving their means of linking those skills and capacities to the world market " (Reich 1992:8).

2. Ce losange se composait de l'état des facteurs de production (dont la main-d'œuvre), l'état de la demande, les activités liées et de soutien et, enfin, de la stratégie de l'entreprise, sa structure et la rivalité. Rappelons, au passage, l'influence de Porter sur la définition de la stratégie québécoise des "grappes * industrielles en 1991, concept promu par cet auteur dans ses travaux. 
exemple : une forte concurrence, une demande exigeante ou la politique publique ${ }^{3}$ ).

Ajoutons aussi, en matière d'influences extérieures sur le Québec, que le rôle stratégique accordé à la main-d'œuvre avait déjà été souligné explicitement dans des travaux portant sur l'adaptation de l'économie canadienne dont le Rapport du Conseil consultatif sur l'adaptation (Rapport DeGrandpré) (1989), l'Énoncé de politique de main-d'œuvre du gouvernement fédéral Le nouveau mode d'emploi, une stratégie de mise en valeur de la maind'œuvre (Emploi et Immigration Canada 1989) et Les chemins de la compétence, Éducation et formation professionnelle au Canada (Conseil économique du Canada 1992).

\section{Un diagnostic inquiétant au tournant de la décennie 90}

En deuxième lieu, le diagnostic posé dans l'Énoncé québécois quant à l'état de la main-d'œuvre et du système de formation professionnelle révélait l'ampleur des correctifs à apporter. Prenant acte des transformations structurelles de l'économie québécoise et de leurs incidences sur le marché du travail, particulièrement quant à la part décroissante des emplois exigeant peu de qualifications, l'Énoncé faisait état à la fois des pénuries de maind'œuvre qualifiée dans certains secteurs, métiers spécialisés ou professions et, à la fois, de la propension au chômage plus élevée chez la main-d'œuvre peu qualifiée. Le marché du travail de l'avenir réserverait d'ailleurs de moins en moins de place à cette dernière catégorie de main-d'œuvre. Le défi de l'adaptation de la main-d'œuvre et du développement des qualifications était sans équivoque pour le développement économique du Québec. À cet égard, le système de formation professionnelle ne semblait tout simplement pas répondre aux exigences modernes pour relever ce défi en regard de la main-d'œuvre. Bien que les efforts publics pour moderniser notre système d'éducation avaient rapporté des fruits quant au rattrapage à effectuer en matière de scolarité de base de la population, des déficits importants persistaient, en témoignent les quelques éléments suivants.

Du côté des politiques de main-d'œuvre, l'accent avait essentiellement été mis sur ce que l'on qualifiait de mesures passives, une faible part des budgets (estimée à quelque $10 \%$ ) étant allouée à des mesures dites actives

3. Sur la politique publique, Porter souligne : "La meilleure façon d'apprécier l'importance de la politique publique consiste à examiner son incidence sur chacun des quatre déterminants de l'avantage concurrentiel composant le losange. On analyse le rôle de l'État en examinant ses effets sur la création de facteurs ainsi que les objectifs et le comportement des particuliers et des entreprises, son rôle d'acheteur de biens et de services, et l'influence qu'il exerce sur le contexte concurrentiel par le biais de la politique de la concurrence, de la réglementation et de sa participation au capital de certaines entreprises " (Porter 1991 : 29). 
favorisant l'adaptation de la main-d'œuvre. Quant à la gestion gouvernementale des programmes et mesures destinées à la formation, on évoquait la complexité, la lourdeur, la confusion engendrée auprès des destinataires de la formation, la double administration fédérale/provinciale, le tout attestant que l'État reconnaissait les limites du système bureaucratique de gestion de la formation. À cet égard, soulignons que les acteurs québécois du marché du travail appuyaient la revendication de ramener au Québec les pouvoirs en matière de main-d'œuvre et de formation considérant que la politique publique gagnerait en efficacité en se rapprochant du niveau d'action des entreprises et de la main-d'œuvre. Par ailleurs, l'Énoncé révélait aussi un faible engagement des employeurs et une gestion peu innovatrice à l'égard de la formation de la main-d'œuvre et ce, surtout chez les petites et moyennes entreprises. Quant à la main-d'œuvre peu qualifiée, elle était également faiblement engagée dans des mesures de formation et peu encline à le faire. Du côté des pratiques conjointes, on identifiait un faible niveau de partenariat dans l'entreprise et en dehors de l'entreprise dans le domaine de la formation. La formation n'était ainsi que marginalement considérée comme un objet de négociation collective, les expériences de concertation sectorielle faisaient figure d'exception (le $\mathrm{CAMAQ}^{4}$ étant pratiquement la seule expérience établie) et, enfin, la seule structure institutionnelle de l'époque s'adressant à la formation, soit les Commissions de formation professionnelle datant de 1969, ne semblait pas jouer un rôle suffisamment actif dans le développement du partenariat.

Enfin, quant à l'articulation entre le système d'éducation/formation et le marché du travail, on décelait plusieurs lacunes majeures. Le Québec connaissait un taux d'analphabétisme fonctionnel élevé (quelque $25 \%$ de la population adulte), le décrochage scolaire oscillait autour de $35 \%$ au niveau secondaire et on observait une chute continue des inscriptions en formation professionnelle tant au niveau secondaire qu'au niveau collégial. Quant à des pratiques de collaboration entre les entreprises et le milieu de l'éducation, du type stages professionnels ou régime d'apprentissage, elles étaient également marginales, essentiellement concentrées autour de quelques métiers réglementés.

$\mathrm{Vu}$ dans son ensemble, le système de formation professionnelle du Québec affichait des faiblesses à tous les niveaux. Les politiques publiques étaient défaillantes, désarticulées sur le plan intra et intergouvernemental ; la structure institutionnelle de formation faisait état d'un clivage quant aux pouvoirs et aux cultures des divers acteurs, la gestion de l'offre de formation

4. Le Centre d'adaptation de la main-d'œuvre aérospatiale au Québec a été formellement créé en 1983 par les employeurs membres de l'Association des industries aérospatiales du Canada et les syndicats de ce secteur, avec l'appui des deux paliers de gouvernement. Des activités préalables à cette création avaient cependant déjà débuté en 1978. 
étant le propre des réseaux publics d'éducation alors que les acteurs " privés " du marché du travail agissaient essentiellement dans une structure de demandeurs de la formation (les CFP) ; l'environnement législatif spécifique à la formation professionnelle n'était pas directif et n'avait pas été modemisé depuis la Loi sur la formation et la qualification professionnelles de la main-d'œeuvre de 1969, considérée elle-même inachevée dans son essence; les comportements (préférences, habitudes) des employeurs et de la main-d'œuvre ne révélaient souvent qu'un faible engagement envers la formation professionnelle continue ${ }^{5}$; les pratiques conjointes des acteurs du marché du travail étaient peu répandues dans l'entreprise et en dehors de celle-ci. Il n'y avait donc pas véritablement de " culture de la formation " au Québec, ni quant à la communauté des pratiques des acteurs, ni quant à l'articulation institutionnelle que ce concept implique. L'Énoncé de politique de 1991 conduisait alors à une conclusion importante relevant de l'observation des expériences étrangères à ce moment : une culture commune de la formation ne se décrète pas, ne s'importe pas, elle est une construction sociale graduelle qui nécessite une participation active et un engagement de tous les acteurs, agissant à tous les niveaux de ce qui constitue un système de formation professionnelle. Pour y parvenir, les actions devaient s'insérer dans une vision stratégique commune et partagée par les divers acteurs québécois.

\section{La stratégie québécoise de développement de la main-d'œuvre: une réponse politique et économique}

On peut résumer la stratégie mise de l'avant initialement dans l'Énoncé de 1991 par les principaux éléments suivants :

- Recentrer graduellement les mesures de développement de la maind'œuvre, des mesures dites " passives " vers les mesures dites " actives ";

- Opérer une gestion unifiée de l'ensemble des programmes de maind'œuvre en rapatriant au Québec tous les budgets fédéraux consacrés à la main-d'œuvre ;

- S'orienter vers une prise en charge du développement de la main-d'œuvre par les acteurs organisés du marché du travail, les entreprises et les individus eux-mêmes;

5. Rappelons cependant que le comportement observé dans les grandes entreprises était généralement plus favorable à l'égard de la formation de la main-d'œuvre. Par ailleurs, soulignons que les organisations syndicales québécoises revendiquaient depuis déjà plusieurs années une intervention plus structurante de la part de l'État à l'égard de l'enjeu de la formation professionnelle continue. Voir à cet effet l'analyse que nous en avons faite (Charest 1998a). 
- Mettre en place une structure permanente de concertation entre les acteurs patronaux, syndicaux et le gouvernement en créant une nouvelle institution publique, soit la Société québécoise de développement de la main-d'œuvre (SQDM) ;

- Décentraliser la politique de développement de la main-d'œuvre vers les niveaux sectoriel et régional (notamment par la création de comités sectoriels de main-d'œuvre et par les directions régionales de la SQDM) ;

- Articuler plus étroitement le milieu de l'éducation et le marché du travail (revaloriser la formation professionnelle, décloisonner les institutions d'enseignement, développer le régime d'apprentissage et instaurer un régime de reconnaissance des acquis professionnels).

Dans le prolongement de cette stratégie, l'État québécois adopta en 1995 une mesure plus coercitive soit la Loi favorisant le développement de la formation de la main-d'œuvre, connue sous le nom de loi 90 ou loi du $1 \% 6$. Celle-ci obligeait les employeurs ayant une masse salariale supérieure à $250000 \$$ à verser un minimum de $1 \%$ de cette masse sur une base annuelle pour des mesures liées à la formation dans l'entreprise, à un organisme collecteur (tel qu'un comité sectoriel) ou, à défaut, dans un nouveau fonds, le Fonds national de formation de la main-d'œuvre. Cette loi disposait pour l'essentiel du crédit d'impôt à la formation introduit quelques années plus tôt, qui demeurait en vigueur jusqu'à la fin de 1998 pour les entreprises non assujetties à la loi et, au-delà de cette date, pour les seules fins du régime d'apprentissage, du régime de qualification et des stages en entreprise visant notamment des personnes engagées dans des programmes d'employabilité (Emploi-Québec 1998: 15, 40).

Pour compléter ce tour d'horizon de la stratégie gouvernementale des années 90, ajoutons deux autres décisions d'importance. En 1997, le gouvernement adopta un projet de loi faisant disparaitre la SQDM, recentralisant ainsi les pouvoirs décisionnels au sein de l'appareil ministériel (sous l'appellation d'Emploi-Québec, constitué en unité autonome de services au sein du ministère de l'Emploi et de la Solidarité) et reléguant les partenaires du

6. Rappelons que dès 1980, la Commission mise sur pied par le gouvernement du Québec pour étudier la formation des adultes, connue sous le nom de Commission Jean, recommandait, entre autres, que chaque entreprise ait l'obligation de consacrer 1,5\% de sa masse salariale à la formation de sa main-d'œuvre, que l'on crée un comité de formation paritaire dans chaque entreprise privée ou établissement public et que l'on donne à un seul organisme central la responsabilité de la formation de la main-d'œuvre au Québec (voir Commission d'étude sur la formation des adultes 1982 : chap. 2). Dans le même sens, les grandes organisations syndicales québécoises revendiquaient depuis les années 80 une loi obligeant les employeurs à consacrer un certain pourcentage de la masse salariale à la formation soit directement dans leur entreprise, soit dans un fonds provincial ( $1 \%$ à $2 \%$ était suggéré). 
marché du travail dans une nouvelle institution consultative la Commission des partenaires du marché du travail. L'autre décision fut l'adoption d'une autre loi en 1997 qui modifiait la loi 90, afin d'introduire formellement un régime d'apprentissage visant à développer la formation en entreprise par le biais d'une collaboration étroite entre les employeurs et les établissements d'enseignement.

Revenons maintenant plus précisément à la question initiale, soit celle des influences que l'on peut dégager en regard des orientations adoptées au Québec dans la modernisation de sa stratégie de formation professionnelle. Quelques remarques s'imposent déjà à ce stade-ci de l'analyse. Nous les regroupons autour de ce que nous nommons une réponse politique, puis une réponse économique.

L'histoire des relations entre le Québec et le fédéral dans le dossier des politiques de main-d'œuvre représente en soi un ensemble de jeux politiques où s'entremêlent au fil des décennies des stratégies d'occupation et de préservation des champs de compétences en ce domaine (Bélanger 1989; Poulin Simon et Carroll 1991 ; Paquet 1993). L'Énoncé de politique de 1991 n'y fait pas exception. Rappelons qu'il s'inscrivait dans la foulée de l'énoncé présenté par le gouvernement fédéral en 1989 et dans la foulée de la Stratégie d'adaptation et de mise en valeur des ressources humaines qu'annoncèrent l'ensemble des provinces canadiennes, dont le Québec, à l'automne 1989 (MMSRFP 1989). Le partenariat des divers paliers de gouvernement et des acteurs du secteur privé y était énoncé clairement. Dès le début de 1991, le gouvernement fédéral répondait à la consultation menée par le Centre canadien du marché du travail et de la productivité sur l'énoncé de politique en annonçant la création de la Commission canadienne de mise en valeur de la main-d'œuvre (CCMMO), organisme consultatif multipartite ayant pour mandat de promouvoir et de soutenir la formation et la mise en valeur de la main-d'œuvre. Cette création donna l'impulsion à la mise sur pied d'un équivalent dans plusieurs provinces?

De plus, rappelons que la stratégie fédérale de mise en valeur de la maind'œuvre s'engageait notamment dans la voie des initiatives de concertation sectorielle bipartite sur le modèle du CAMAQ et du CCCES (Conseil canadien du commerce et de l'emploi dans la sidérurgie), en prévoyant la création de plusieurs conseils sectoriels canadiens de développement des

7. Entre 1992 et 1994 apparaîtront des commissions dans les provinces suivantes : ColombieBritannique, Saskatchewan, Ontario, Nouveau-Brunswick, Nouvelle-Écosse, Terre-Neuve/ Labrador; le Québec créera la Société québécoise de développement de la main-d'œuvre en 1993. 
ressources humaines ${ }^{8}$. Tel qu'on le rappelle dans l'Énoncé de politique de 1991, le gouvernement du Québec, avec l'appui des différents partenaires du marché du travail, se positionnait face à cette stratégie fédérale en revendiquant officiellement en décembre 1990 le rapatriement de tous les budgets fédéraux relatifs aux programmes concernant la main-d'œuvre du Québec. À cette réponse politique du gouvernement du Québec s'adjoignait la non-participation officielle des différents acteurs québécois aux nouvelles institutions canadiennes destinées à la mise en valeur de la main-d'œuvre (la CCMMO et les conseils sectoriels) et la création au Québec d'institutions de concertation en la matière (d'abord avec la Conférence permanente sur la main-d'œuvre, puis la SQDM et les comités sectoriels de main-d'œuvre).

Très clairement, on peut dégager ici que des influences importantes, bien que non exclusives en regard des orientations du Québec pour le développement de sa main-d'œuvre, provenaient effectivement d'une expérience extérieure, en l'occurrence la mise sur pied de la stratégie fédérale accompagnée de nouvelles institutions canadiennes. La forme institutionnelle que prenait la modernisation de la stratégie québécoise de développement de la main-d'œuvre était en partie une réponse politique ${ }^{9}$. On pourrait certes arguer que le Québec faisait preuve d'un sens de l'innovation plus audacieux en créant une véritable société d'État autonome gérée avec les partenaires du marché du travail, soit la SQDM, plutôt qu'une simple structure consultative, ce qu'était la CCMMO. Mais reconnaissons que cette distinction n'aura été que bien éphémère, considérant l'abolition rapide de la SQDM $^{10}$.

D'autre part, on peut dire que la nouvelle stratégie québécoise de développement de la main-d'œuvre des années 90, incluant l'introduction de la loi du $1 \%$, était également une réponse économique face à l'enjeu de la formation professionnelle continue. À ce sujet, l'analyse que nous proposons ici n'est pas toujours appuyée sur un discours explicite de l'État et nous procédons donc par inférence en dégageant une formulation davantage théorique à partir de la lecture des faits et actions de l'État recensés précédemment. Nous proposons l'analyse suivante.

8. En 1992, on en comptait déjà une dizaine et, en 1995, 20 conseils canadiens étaient en opération soutenus par un financement important de Développement des ressources humaines Canada (DRHC 1995).

9. D'autres chercheurs partagent cette analyse. Voir, en particulier, Sharpe et Haddow (1997 : 8).

10. Cette abolition suivait de peu d'ailleurs une décision similaire du gouvernement de l'Ontario d'abolir sa propre institution de concertation dans le domaine du développement de la main-d'cuvre, fort récente également, le Ontario Training Advisory Board, abolition effective en avril 1996. En conséquence, les fonctions dévolues à cette institution furent rapatriées au sein de l'appareil gouvememental. 
L'État québécois a situé l'enjeu de la formation professionnelle continue en partie dans la sphère des biens publics, ce qui est le cas spécifique de la formation dite "qualifiante ou transférable ", soit celle qui améliore les compétences de la main-d'œuvre. Cette formation est déterminante pour assurer le développement continu de l'employabilité de la main-d'œuvre et donc pour assurer sa mobilité, devenues des impératifs dans le contexte d'un marché du travail plus fluide que caractérisent les transformations économiques en cours $^{11}$. Parallèlement à cette formation qualifiante ou transférable, l'État reconnaissait aussi l'importance de la formation spécifique à l'entreprise, celle-ci étant toutefois considérée comme une responsabilité propre à l'entreprise qui bénéficiera en exclusivité de cette formation. Cette distinction entre la formation qualifiante et la formation spécifique se retrouve ainsi notamment dans la volonté du législateur qui indique à l'entreprise que les dépenses de formation admissibles à l'obligation du $1 \%$ doivent correspondre à une amélioration des compétences de sa maind'œuvre (voir les précisions sur le type de dépenses admissibles : EmploiQuébec 1998 : section 1).

Ce caractère de bien public de la formation transférable de la maind'œuvre a souvent été invoqué dans la littérature économique à l'instar des travaux de la théorie du capital humain développée par Gary Becker dès le début des années 60 . On peut la présenter brièvement de la façon suivante. Les employeurs ne pouvant être assurés de récupérer leur investissement dans la formation dite transférable de leur main-d'œuvre (celle-ci pouvant quitter l'emploi, notamment parce que recrutée par un autre employeur qui n'aurait pas eu à payer pour la formation), ils auront tendance à se limiter au financement d'une formation spécifique (pointue), car non transférable, ce qui leur donnera davantage l'assurance d'être les seuls employeurs à pouvoir profiter de cette formation. On assistera alors à un problème de sousfinancement et de sous-production de la formation à caractère générale, c'est-à-dire celle qui assure la mobilité et la réadaptation de la main-d'œuvre, à moins que l'État n'en finance la totalité (ce qui nécessiterait tout de même qu'il connaisse les besoins individuels de formation et qu'il détermine aussi le mode de financement collectif de ce " service public ") ou que les individus ne le fassent eux-mêmes (ce qui soulèvera généralement un problème réel de capacité de financement de leur part, surtout chez la main-d'œuvre moins spécialisée ou dont les coûts de formation sont élevés). En d'autres termes, le mécanisme du marché n'est pas adéquat pour assurer une solution optimale au financement de cette formation qualifiante ou transférable,

11. On peut lire à cet effet dans le Guide d'Emploi-Québec : « L'objectif de cette Loi est d'améliorer la qualification de la main-d'œuvre, favorisant ainsi l'emploi, l'adaptation et l'insertion en emploi, ainsi que la mobilité de la main-d'œuvre " (Emploi-Québec 1998 : 5). 
surtout lorsqu'il y a un très grand nombre d'acteurs individuels, ce qui est le cas du marché du travail et de la structure industrielle québécoise où prédominent les petites et moyennes entreprises. La solution à un tel problème passe alors par une forme coordonnée de production et de financement conjoint où chacun saura que tous contribuent au coût de production de la formation qualifiante et que tous peuvent potentiellement en tirer avantage.

L'État joue traditionnellement un rôle important dans ce processus collectif de gestion des biens publics, ce qui peut prendre plusieurs formes concrètes dont, ici, la loi du $1 \%$ qui assure une obligation commune de participer au coût de la formation qualifiante. Cette gestion collective ou coordonnée de la production de la formation a aussi pris des formes institutionnelles supportées par l'État et regroupant les acteurs du marché du travail au Québec, ceux-ci étant considérés les mieux placés pour déterminer leurs besoins et les solutions face à la formation transférable. Outre l'institution centrale (la SQDM), les institutions de niveau intermédiaire, que sont les structures régionales ou les comités sectoriels de main-d'œuvre, sont alors introduites pour permettre aux acteurs d'une région donnée ou d'un secteur donné d'élaborer des objectifs/besoins communs en matière de formation de la main-d'œuvre et de définir des solutions communes.

Dans une telle réponse économique face à l'enjeu de la formation, l'État assure ainsi une partie des coûts de production de la formation (par les institutions publiques d'éducation et de formation continue, par le support financier à la main-d'œuvre en réinsertion et par le soutien des formes institutionnelles de concertation), il crée un environnement législatif et institutionnel mettant les acteurs privés devant les mêmes responsabilités et possibilités et, enfin, il fait payer les acteurs, en même temps qu'il cherche à modifier leur comportement à moyen et long terme. À cet égard, les institutions exercent une fonction importante quant à la production et à la circulation d'informations nécessaires à la prise de décisions des acteurs du marché du travail (par exemple : en matière de prévision des besoins de main-d'œuvre, de définition et d'attestation des qualifications professionnelles, de disponibilité des programmes et ressources en matière de formation ou d'agrément des formateurs). Sur le plan de l'efficacité économique, les institutions contribuent ainsi à réduire l'incertitude chez les acteurs et à réduire les coûts de transaction que chacun aurait à supporter en l'absence de telles institutions, ce que suggérait déjà en substance dès les années 1930 un des économistes fondateurs du courant institutionnaliste, John R. Commons (1934).

Il s'agit donc bien d'une réponse économique en ce sens qu'elle repose sur des fondements théoriques de l'économie publique et de l'approche institutionnaliste qui démontrent que le libre marché n'est pas toujours la meilleure solution à un problème de rareté et de besoins, les institutions ou 
formes collectives de production étant un substitut efficace dans certaines conditions (voir, à cet effet, les analyses de Frey 1985 et Olson 1978 pour une analyse dynamique en économie publique et les travaux de Commons 1934 et 1950 pour les fondements de l'approche institutionnaliste). Une telle solution institutionnelle nécessite toutefois un certain temps pour produire des résultats. Les acteurs doivent apprendre à travailler ensemble y compris entre firmes concurrentes (développement de rapports de confiance), souvent dans le cadre de nouvelles règles (tel que le fonctionnement consensuel et paritaire), se confrontant à des cultures diverses (celle des gestionnaires de l'entreprise, des organisations syndicales, de l'appareil d'État, des institutions publiques d'enseignement), le tout devant conduire à une logique unifiée de résultats (répondre aux besoins de la main-d'œuvre et des entreprises en matière de formation). Il n'y a aucune raison ni théorique, ni empirique, pour qu'un tel processus produise des résultats spontanés, pas plus d'ailleurs que le libre jeu du marché n'en produit lui-même. Malheureusement, les acteurs, y compris l'État, revendiquent souvent une rapidité d'intervention à de tels mécanismes institutionnels et une efficacité en termes de résultats, que le fonctionnement du libre marché n'a pas réussi à atteindre, voire ne pourrait atteindre ${ }^{12}$. L'expérience internationale est aussi probante à cet égard ; la solution à la problématique de la formation n'est ni simple, ni arrêtée dans une forme précise idéale. C'est un tel regard international que nous proposons maintenant pour situer l'expérience québécoise.

\section{LE SYSTĖME QUÉBÉCOIS DE FORMATION PAR RAPPORT À D'AUTRES CONFIGURATIONS NATIONALES}

Même si l'on évoque que la mondialisation se traduit par des pressions de plus en plus similaires sur les pays, les adaptations dans les systèmes nationaux de formation demeurent dominées par des stratégies définies plus ou moins explicitement par les acteurs nationaux ${ }^{13}$. L'analyse comparative

12. La distinction faite ici entre " mécanismes institutionnels " et " libre jeu du marché " est utile pour des fins pédagogiques en ce qu'elle permet de caractériser deux formes contemporaines importantes du marché du travail. La réalité est cependant le fait d'une forte imbrication de ces deux formes d'organisation, le marché n'opérant jamais sans encadrement institutionnel et les institutions étant en situation d'interdépendance avec le marché. Le rapport entre " institutions " et " marché " ne se résume donc certes pas à une stricte division/opposition entre ces deux formes, ce qui est sous-entendu dans nombre d'analyses assises sur une soi-disant dichotomie "État/marché ". Voir à ce sujet les contributions récentes présentées par plusieurs auteurs dans Hollingsworth et Boyer (1997); appliquée spécifiquement au sujet de la formation, voir l'analyse de Sergio Bruno (1998).

13. Sur le plan théorique, on pourra se référer à cet égard aux travaux de Douglas North (1990) qui a relevé les raisons faisant que des pays s'inscrivaient dans certaines trajectoires 
permet de révéler les spécificités nationales de ces systèmes et de les appréhender comme un produit original historiquement construit par les acteurs influents du système de relations industrielles, voire du système social. Mais l'analyse comparative permet aussi de dégager les traits communs aux différents systèmes nationaux et de déceler certaines lignes directrices dans les transformations. Nous proposons ici un tel exercice sur la base d'une typologie des systèmes nationaux de formation et d'études de cas nationaux pour tenter de situer le système québécois, plus particulièrement quant à ses récentes orientations.

\section{Typologie des systèmes nationaux de formation}

Parmi les analyses comparatives internationales des systèmes nationaux de formation, deux études nous apparaissent particulièrement intéressantes, notamment quant à la vision d'ensemble qu'elles suggèrent. D'abord, Lisa M. Lynch (1994) a proposé une typologie de ces systèmes nationaux permettant de caractériser le cas d'une dizaine de pays au début de la décennie 90, dont le Canada. Plus récemment, le BIT (1998) a aussi suggéré une telle typologie où l'on ne retrouve pas cependant le cas du Canada, ni celui de la France ou de l'Australie, mais où l'on retrouve les cas de plusieurs économies en développement. En croisant les informations présentées dans ces deux typologies et en insérant certaines variantes, notamment en regard de la situation du Québec, l'on obtient une présentation d'ensemble intéressante pour les fins de l'analyse comparative ici visée. C'est une telle adaptation de ces deux propositions que l'on retrouve dans le tableau 1.

Bien sûr, comme toute typologie, ce tableau présente une vision incomplète de la réalité des différents systèmes nationaux notamment parce qu'il ne permet pas d'y inscrire toutes les spécificités de chacun de ces systèmes et aussi parce qu'il catégorise ces systèmes en nivelant les différences sectorielles, régionales ou celles relatives à la taille des entreprises. Néanmoins, cette typologie est utile pour saisir les traits marquants de chacun des cas

institutionnelles nationales, dont il peut être difficile de sortir, ce qu'il nomme une situation de "path dependency ». Pour sa part, Charles F. Sabel (1995) a suggéré que la démarche d'adaptation nationale des acteurs face aux pressions de l'environnement mondial n'était pas décrite ou construite a priori par ces acteurs, mais plutôt le produit d'une démarche empirique que l'on peut apparenter à un processus " essais/erreurs " qu'il qualifie de «bootstrapping reform ". Enfin, mentionnons aussi les analyses de Geoffrey M. Hodgson $(1988,1998)$ qui soutient également cette idée générale que les institutions économiques sont une construction des acteurs sociaux, qu'elles renforcent leurs pratiques et leurs habitudes, tout en étant influencées par ces dernières. Il suggère alors d'analyser ce qu'il nomme les systèmes macroéconomiques (dans notre cas ici, les systèmes nationaux de formation) : « ... by examining patterns and regularities of human behavior, expecting to find a great deal of imitation, inertia, lock-in and cumulative causation " $(1998: 171)$. 


\section{TABLEAU 1}

\section{Typologie des systèmes nationaux de formation dans les années 90}

\begin{tabular}{lll}
\hline \multicolumn{1}{c}{ Systèmes } & \multicolumn{1}{c}{ Pays } & \multicolumn{1}{c}{ Caractéristiques } \\
\hline Système basé sur la & Allemagne, Suède, Autriche, & Régulation conjointe (tripartite) \\
coopération & Suisse, Norvège, Pays-Bas, & de la formation; fort röle des \\
État/entreprises/syndicats & Royaume-Uni (avant 1980), & institutions intermédiaires; coo- \\
& plusieurs pays d'Amérique latine & pération école/entreprise; par- \\
& & tage des efforts financiers entre \\
& les acteurs; fortes pressions/ \\
& valorisation à l'égard de la for- \\
& mation professionnelle.
\end{tabular}

- avec coercition liée à une obligation légale d'investir en formation

Système basé sur l'entreprise

- avec forte stabilité du lien d'emploi dans l'entreprise

- avec démarche volontaire de l'entreprise

Système basé sur l'État

- axé sur la demande de formation

- axé sur la gestion de l'offre de formation
France, Australie (1990-94), Québec (1995- )

Japon

États-Unis, Royaume-Uni (1980-), Australie (1994-), Canada (et Québec avant 1995)

Hong-Kong, République de Corée, Singapour, Taïwan, Chine

Pays d'Europe de l'Est ; plusieurs économies en développement (en particulier Asie et Afrique)
Utilisation de la loi comme levier pour développer la culture de la formation en entreprise, pour induire le développement d'institutions intermédiaires et pour favoriser un rapprochement entre offreurs et demandeurs de formation.

Effort de formation important dans les entreprises, fort lien formation/organisation du travail/ marché du travail interne à l'entreprise/rémunération (stabilité du lien d'emploi et promotion interne), forte homogénéité de la formation de base de la m-o.

Autonomie des individus et des entreprises en regard de l'effort de formation ; faible coordination des acteurs ; faible coopération école/entreprise ; multiplicité des lieux de formation (public, privé, entreprise); formation en emploi surtout spécifique à l'entreprise; faibles pressions de l'État.

Rôle central de l'État dans l'ajustement de l'offre à la demande de qualification provenant des entreprises privées (gestion centralisée pro-active).

Rôle central de l'État dans la formation surtout axé sur la gestion de l'offre de formation; faibles pressions sur les employeurs.

Sources: Adaptation des typologies suggérées par Lynch (1994) et BIT (1998) avec ajouts pour le Québec et l'Australie. 
nationaux ${ }^{14}$. On y retrouve une catégorisation des systèmes de formation selon qu'ils reposent davantage sur la coopération, davantage sur l'entreprise avec différentes modalités externes ou internes à celle-ci et, enfin, davantage sur une gestion centralisée de l'État pour répondre à la demande du secteur privé ou dans le cadre d'une planification offre/demande publiques de l'emploi et des qualifications. Pour les fins de l'analyse du cas québécois ici considéré, limitons-nous à l'examen de quelques-uns de ces systèmes nationaux.

Dans le premier groupe de pays (système basé sur la coopération), l'Allemagne est sans doute le modèle le plus connu, notamment en raison de son système d'apprentissage ("système dual ") qui constitue le pivot de la relation école/entreprise, ainsi qu'il constitue l'assise de la formation professionnelle des jeunes et de leur accès au marché du travail (Maurice 1993 ; Bosch 1993 ; Soskice 1994). Les pays de ce groupe partagent une vision de la gestion de la formation qui s'appuie sur ce que nous nommons ici une régulation conjointe. Cela renvoie à un partage des responsabilités entre les acteurs, à une décentralisation des pouvoirs de l'État vers les autres acteurs et vers les institutions intermédiaires (chambres de commerce, fédérations syndicales, structures sectorielles ou régionales), à une étroite collaboration entre le système public d'éducation et les entreprises, et à un partage des coûts de la formation entre les différents acteurs ${ }^{15}$. Ce type de système est ainsi construit sur de fortes imbrications entre les besoins et les logiques respectives des acteurs qui transitent par un réseau d'institutions et qui donnent lieu à des négociations continuelles (Maurice 1993; Locke et Jacoby 1997).

Ce système de régulation conjointe, comme tous les autres, demeure perméable aux pressions de l'environnement et il reflète la dynamique des rapports entre les acteurs qui s'y retrouvent. À titre d'exemple, les entreprises allemandes sont aussi influencées par la tendance à la réduction des coûts de production, incluant les coûts de main-d'œuvre, ce qui en a conduit plusieurs à réduire leurs dépenses liées à la formation, dont celles liées au régime d'apprentissage, réduisant ainsi la disponibilité des places en entreprise pour ce régime (BIT 1998 : 74 ; Büchtemann et Soloff 1998: 17).

14. L'analyse de ces spécificités nationales ne sera que très brève ici, l'objet étant davantage de situer le Québec par rapport aux diverses configurations nationales. Voir le chapitre 3 du rapport du BIT (1998) pour une analyse détaillée de ces spécificités.

15. Notons ici que les pays d'Amérique latine ont un système coopératif qui se distingue des cas européens. Essentiellement, il consiste en la mise sur pied d'établissements de formation professionnelle distincts des autres établissements scolaires assurant la formation générale et financés par des cotisations sur les masses salariales des employeurs (de $1 \%$ à $2 \%$ ). Selon les pays, la gestion en est plus ou moins partagée entre les divers acteurs (État, employeurs, syndicats) (BIT $1998: 75-77$ ). 
On peut aussi dire que le processus de négociation entre acteurs sociaux occasionne une certaine lourdeur et lenteur dans l'adaptation entre les programmes de formation et les besoins de l'industrie (Bosch 1993). Le cadre volontaire de ce système fait également que plusieurs entreprises choisissent de ne pas participer à un tel système de régulation conjointe de l'effort de formation (avant la réunification, on estimait que seulement $20 \%$ des entreprises étaient formellement engagées dans le système de formation en Allemagne de l'Ouest) (Locke et Jacoby 1997 : 36). Ce type de système n'est pas à l'abri non plus des changements dans les pratiques et les orientations de ses acteurs (voir par exemple les transformations importantes en Suède depuis les années 90 dans les mécanismes tripartites de régulation conjointe). Toutefois, malgré ces pressions, on observe une certaine stabilité de ces systèmes nationaux parce qu'ils assurent généralement aux employeurs l'accès à une main-d'œuvre qualifiée, parce qu'ils assurent un bon taux de placement aux jeunes et aussi parce qu'ils contribuent au développement d'institutions et de réseaux qui améliorent l'efficacité du fonctionnement du marché du travail.

Nous avons introduit dans ces systèmes axés sur la coopération, une variante où la recherche du développement d'une culture de la formation dans les milieux de travail est supportée par une loi spécifique qui impose une obligation d'effort (pas nécessairement une obligation de résultats précis) à certaines entreprises sur le plan des ressources monétaires à consacrer à la formation. Cette approche utilise en fait l'encadrement légal, eu égard à l'investissement obligatoire en formation, comme un levier pour changer les habitudes des gestionnaires et des salariés à l'égard de la formation et comme un moyen d'assurer le financement collectif de la formation transférable, considérée comme un bien public. La loi devient un moyen coercitif de financement collectif de la formation et de développement graduel d'une culture de la formation, en révélant l'enjeu public qu'est la formation qualifiante, tout en laissant généralement une bonne marge de manœuvre à l'entreprise quant aux moyens à adopter (allant du " on-thejob training " à la formation externe par le biais des institutions publiques).

La France expérimente cette approche depuis l'adoption d'une loi obligeant un effort de formation en 1971. Les entreprises sont soumises à deux " taxes ": une taxe d'apprentissage $(0,5 \%$ de la masse salariale) et une taxe de formation, dont le pourcentage est passé graduellement de $0,8 \%$ à $1,5 \%$ de la masse salariale des entreprises ayant au moins dix salariés $(0,25 \%$ pour les moins de dix salariés). En fait, il s'agit d'une taxe au sens où les entreprises, qui ne rencontrent pas dans leurs propres dépenses de formation l'effort total exigé par la loi, devront payer l'équivalent sous la forme d'une taxe à des fonds, à des organismes destinés à la formation ou à l'État. Ces pourcentages obligatoires sont divisés en catégories de dépenses : une partie doit 
être consacrée à une formation qualifiante interne à l'entreprise $(0,9 \%)$, une autre partie doit servir à financer les activités des programmes de formation en alternance pour les jeunes $(0,4 \%)$, et une autre sert à financer les fonds pour le congé individuel de formation $(0,2 \%)^{16}$. Une distribution précise est également prévue pour le 0,5\% qui vise l'apprentissage (Gasskov 1998; BIT 1998).

L'Australie s'est inspirée de l'approche française en adoptant un tel mécanisme législatif en 1990 qui imposait une obligation de consacrer un minimum de $1,0 \%$ (puis de $1,5 \%$ en 1992-93) de la masse salariale à la formation dans les entreprises (lorsque cette masse salariale est de 200000 dollars australiens et plus). À peine quatre ans après son entrée en vigueur, et malgré un bilan relativement positif, le gouvernement Australien suspendait l'application de cette loi en 1994, dans le contexte de transformations importantes au sein du système de relations industrielles et sur le plan de l'orientation de la politique économique. L'arrivée au pouvoir d'un nouveau gouvernement en 1996 conduisit à une nouvelle stratégie de formation plus libérale, davantage centrée sur une approche volontaire de l'entreprise (Fraser 1996; Ewer 1998). Mentionnons aussi au passage que ce modèle s'appuyant sur une loi coercitive a également été envisagé aux États-Unis au début des années 90 (Lynch 1994 : 5). Notamment, le Parti démocrate de Bill Clinton en avait fait une proposition de campagne présidentielle en indiquant que toutes les entreprises de 50 salariés et plus auraient l'obligation de consacrer un minimum de $1,5 \%$ de leur masse salariale à la formation, sous peine de verser l'équivalent à un fonds servant à mettre sur pied des centres régionaux de formation. Suite aux pressions exercées par les employeurs, l'administration Clinton abandonna cette idée une fois les élections passées (Bernstein et Magnusson 1993).

Les évaluations sont encore imprécises quant aux effets d'une telle approche d'encadrement législatif de la formation en entreprise. D'une part, le cas québécois est très récent et l'Australie a changé sa politique après

16. Soulignons ici qu'outre la loi imposant des obligations aux entreprises, la France a également adopté une mesure permettant aux salariés du secteur privé de se prévaloir d'un congé individuel de formation. Ce congé est géré par des organismes paritaires dont le financement provient d'une partie $(0,2 \%)$ de la contribution obligatoire de $1,5 \%$ des employeurs. Pendant la formation, les salariés reçoivent de $80 \%$ à $90 \%$ de leur salaire ( $60 \%$ au cours de la deuxième année si la formation dépasse un an ou 1200 heures). C'est l'organisme paritaire qui rembourse à l'employeur le salaire occasionné par le congé. L'État contribue aussi au financement des organismes paritaires, notamment pour qu'ils puissent assumer le remplacement du salaire pour la main-d'œuvre provenant des entreprises de moins de dix salariés. Les demandes de formation sont gérées par l'organisme paritaire qui accepte en moyenne $70 \%$ des demandes par année (soit environ 20000 demandes acceptées annuellement). Le congé annuel moyen dure 850 heures (environ 24 semaines) (Gasskov 1998: 35-36). 
une courte expérience seulement, dans le cadre d'une modification de l'ensemble de sa politique économique. D'autre part, même dans une expérience plus longue comme le cas français, il y a des limites quant à la possibilité d'isoler les effets de la loi sur les résultats en matière de formation par rapport à l'effet d'autres variables de l'environnement des entreprises et des individus, comme il y a des limites aussi à "mesurer "les changements induits. Selon le BIT : "La grande diversité des programmes et des niveaux de formation, et le nombre des entreprises participantes rendent très difficiles le suivi et l'évaluation de la formation. L'évaluation se limite trop souvent à déterminer si les entreprises ont rempli leurs obligations formelles, sans évaluer la qualité, la pertinence et l'équité de la formation "(BIT 1998: 100). D'autres, après avoir relevé que les entreprises françaises ont pratiquement toujours dépassé les seuils minimaux fixés par la loi, suggèrent néanmoins que la loi française a permis « la création d'un marché de la formation solide " référant ainsi à l'aspect structurant que la loi a pu avoir sur l'organisation de la formation en France (Venna 1998 : 60). Ce dernier aspect doit être considéré en soi comme un résultat important si l'on se réfère à l'un des objectifs visés par l'État dans la réponse économique au problème de la formation, telle que nous l'avons présentée précédemment.

Par ailleurs, certaines questions demeurent encore ouvertes. Ainsi, fautil fixer un pourcentage minimal équivalent pour toutes les entreprises peu importe leur secteur d'activité et leur taille? L'État doit-il et peut-il être plus directif quant à la distribution de l'effort de formation selon les différentes catégories de main-d'œuvre dans l'entreprise ? Celle-ci n'aura-t-elle pas toujours tendance à sélectionner la main-d'œuvre qui garantit le meilleur retour sur l'investissement en formation? Enfin, mentionnons que le recours à une loi et l'analyse de ses effets renvoient forcément à la prise en compte des autres actions nécessaires en matière de formation, qu'il s'agisse de l'existence d'un réseau public d'éducation de qualité, de l'existence de politiques publiques visant la réinsertion en emploi, de la mise en action et en réseau d'institutions centrales et intermédiaires ou, encore, de l'engagement des entreprises à l'égard de la formation. Bref, le recours à une loi n'est pas une panacée, c'est un élément d'une stratégie globale et intégrée en faveur du développement de la main-d'œuvre.

Le deuxième groupe de pays est en fait composé de sous-groupes dont le système de formation gravite surtout autour de l'entreprise et des marchés internes du travail. Cela ne signifie pas que le système public d'éducation n'y concoure pas, mais en général l'enseignement professionnel y est peu valorisé, il attire une faible part des jeunes et il est faiblement articulé avec le monde du travail. Le Japon représente un cas particulier où l'entreprise joue un rôle central dans la formation professionnelle continue, 
celle-ci étant étroitement associée à l'organisation du travail, à la stabilité de l'emploi dans le cadre de marchés du travail intemes aux entreprises et à la rémunération des salariés. De même, la distinction entre la formation en milieu scolaire (générale) et la formation en entreprise (technique) fait partie de l'organisation du système de formation. Les caractéristiques de ce modèle sont assez peu communes à la situation québécoise et nous n'en traiterons pas davantage ici (voir Sato 1993 ; Hashimoto 1994). Notons simplement que ce modèle subit des pressions importantes compte tenu de la détérioration de l'économie et des conditions du marché du travail, notamment en regard de la main-d'œuvre moins intégrée dans les marchés internes (à ce sujet, voir l'analyse récente du BIT 1998 : 77-78).

Dans ce modèle basé sur l'entreprise, on retrouve une variante plus " libérale " avec le cas des États-Unis, du Royaume-Uni depuis 1980, de l'Australie depuis 1994 et du Canada (dont le Québec avant l'adoption de la loi du $1 \%$ ). L'effort de formation y est surtout reporté sur les choix des individus avant et pendant leur vie active ; la coordination des acteurs du marché du travail entre eux et avec le milieu de l'éducation est faible ; l'État s'attend à ce que la culture de la formation émane surtout des pressions de la concurrence sur les entreprises et la main-d'œuvre, même s'il en encourage le développement publiquement et à l'aide de certains programmes de subvention.

Les critiques sont assez sévères eu égard à la capacité d'un tel modèle à soutenir le développement d'une culture de la formation et à rehausser les investissements en formation dans les milieux de travail. On constate que ce modèle libéral conduit à une coopération assez faible entre l'école et l'entreprise, à une formation en entreprise essentiellement spécifique à ses besoins (formation dite "pointue") et à une distribution de l'effort de formation dirigée davantage vers les clientèles déjà mieux pourvues en la matière, ce qui conduit à une "polarisation des qualifications " (les moins qualifiés recevant le moins de formation) (BIT 1998 ; Lynch 1994 ; Steedman 1993 ; West, Pennell et Edge 1998 ; Conseil économique du Canada 1992 ; Betcherman, McMullen et Davidman 1998 ; Frazis et al. 1998). L'État demeure alors le gestionnaire de l'adaptation de la main-d'œuvre la moins qualifiée et la plus instable sur le marché du travail avec des résultats mitigés en termes de participation de cette main-d'œuvre aux programmes gouvernementaux et en termes de stabilisation de la situation de celle-ci sur le marché du travail. La situation canadienne relève de ce type de système libéral bien que des recommandations et certaines actions aient cherché à rapprocher les pratiques de formation vers le pôle coopératif et intégrateur décrit précédemment. En fait, comme nous allons le constater, la situation au Canada et au Québec a été influencée par des tendances diverses dans les années 90. 


\section{Les influences extérieures sur le système québécois}

La typologie des systèmes nationaux de formation et la brève description que nous en avons faite permettent de dégager un certain nombre d'influences extérieures ou de références étrangères eu égard aux développements qu'a connus le système québécois de formation au cours des années 90 . Notre analyse conduit à identifier quatre tendances particulières qui ont pu inspirer les développements au Québec. Une tendance vers la régulation conjointe, une tendance vers la décentralisation, une tendance à l'utilisation du cadre législatif et une tendance vers l'autonomie décisionnelle des acteurs de l'entreprise. Ces tendances représentent en quelque sorte des forces d'attraction que l'on peut rattacher à des systèmes différents. Elles ne permettent donc pas de conclure au déplacement du Québec vers un modèle précis de formation professionnelle. Au total, nous considérons que le système québécois est allé dans le sens d'une articulation plus étroite des différents acteurs concernés par la formation, recherchant une structuration des rapports entre ces acteurs, mais tout en gardant un attachement fort à la liberté décisionnelle des acteurs de l'entreprise, voire des individus, à la " manière américaine " qui caractérise à bien des égards la dynamique de notre économie et de notre système de relations industrielles. Élaborons quelque peu sur ces quatre tendances.

La tendance à la régulation conjointe, telle qu'elle a été développée par plusieurs pays d'Europe souvent appelés "modèles néo-corporatistes " et qu'on retrouve dans le système coopératif de notre typologie, a inspiré à notre avis la logique du partenariat et de la concertation entre les acteurs que l'on a retrouvée au Québec au début des années 90 , de même qu'ailleurs au Canada ${ }^{17}$. Celle-ci était bien présente, comme nous l'avons vu précédemment, dans l'Énoncé de 1991, mais aussi dans la stratégie des grappes industrielles du ministère de l'Industrie, du Commerce et de la Technologie dès 1991. Cette idée de régulation conjointe en matière de formation a pris forme concrètement sur le plan institutionnel avec la création de la SQDM en 1993. L'État confiait ainsi des responsabilités importantes et un pouvoir décisionnel stratégique aux acteurs du marché du travail. "La Société est en effet appelée à devenir l'une des grandes organisations efficaces du Québec moderne "pouvait-on lire dans l'Énoncé de politique de maind'œuvre de 1991. Plus récemment, l'adoption en 1997 d'une loi visant la

17. Au niveau fédéral, la CCMMO avait davantage un rôle consultatif mais il s'agissait aussi d'une structure multipartite en matière de développement de la main-d'œuvre à la manière " européenne néo-corporatiste ". L'Ontario Training Advisory Board (OTAB) et les autres organismes provinciaux équivalents suivaient aussi cette tendance. Au début des années 90 , l'heure québécoise, mais aussi l'heure canadienne, étaient à la concertation structurée, organisée, et soi-disant permanente. 
mise en place d'un régime d'apprentissage au Québec s'inscrivait également dans cette tendance que nous qualifions ici de régulation conjointe au sens où l'on cherche par une telle mesure à rapprocher l'école et l'entreprise dans la gestion de la formation professionnelle.

Bien que nous n'analysions pas ici les causes probables (dont les changements de gouvernement) de certains revirements importants dans cette tendance à la régulation conjointe, force nous est de constater que cette tendance, prenant forme dans des structures institutionnelles " nationales " de partage des pouvoirs entre les acteurs, a été bien instable. Nous l'avons déjà souligné, la SQDM n'a duré qu'à peine quatre ans suite à une décision du gouvernement de la remplacer par une nouvelle entité autonome de services (Emploi-Québec) dont on ne connaît pas encore la véritable dynamique, notamment en regard du rôle des acteurs présents à la Commission des partenaires du marché du travail. En Ontario, on a assisté à une véritable recentralisation des pouvoirs suite à la disparition de l'OTAB. Quant à la CCMMO, elle a vu ses budgets et son envergure être fortement diminués seulement trois ans après sa création et, au tournant de 1998-99, les acteurs patronaux se retiraient du CCMMO disant ne plus voir l'utilité d'y siéger. Au Québec, les rôles respectifs de la nouvelle Commission des partenaires et d'Emploi-Québec, ainsi que l'influence réelle des acteurs du marché du travail par rapport aux orientations gouvernementales en matière de maind'œuvre et de formation, nous apparaissent comme des éléments clés à considérer dans l'avenir immédiat pour juger de la tendance au partenariat et à la responsabilisation des acteurs "privés " du marché du travail. Notre analyse des rapports récents entre les différents acteurs et des gestes de l'État (allant de l'abolition de la SQDM, jusqu'au congédiement par la ministre de la présidente de la Commission des partenaires, présidente choisie par les partenaires eux-mêmes) est à l'effet qu'il y a de fortes chances que nous assistions à une recentralisation des pouvoirs décisionnels entre les mains de l'État, confinant les partenaires à un rôle strictement consultatif qui s'écarterait alors de loin des orientations initiales de l'Énoncé de politique de 1991.

La deuxième tendance, celle vers la décentralisation, en a été une d'importance également dans le système de formation québécois des années 90. Cette décentralisation s'inspirait aussi de l'idée de l'engagement des différents acteurs du marché du travail et de l'éducation dans la gestion de la formation, dans la perspective d'assurer une meilleure adéquation entre les besoins des uns et les services des autres. Nous avons également évoqué, dans la première partie de ce texte, que cette décentralisation correspondait à une logique économique de regroupement des acteurs dans des structures intermédiaires afin de répondre à l'enjeu de la formation considérée comme un bien public. Dans le domaine de la formation de la main-d'œuvre, cette décentralisation a pris la voie des structures régionales et des comités 
sectoriels que l'on retrouve aujourd'hui dans quelque vingt-quatre secteurs d'activité économique au Québec. Pour le moment, les études sur les structures sectorielles ou régionales reliées à la formation sont encore peu nombreuses au Québec (voir Bernier et Bilodeau 1997 ; Charest 1998b). Mais l'on constate que cette tendance à la décentralisation s'est poursuivie avec l'introduction plus récente, et toujours en cours actuellement, d'un peu plus d'une centaine de CLD (Centre local de développement) et de CLE (Centre local d'emploi) qui regroupent plusieurs partenaires locaux en matière de développement économique et de développement de la main-d'œuvre.

En termes d'expériences étrangères, on remarque que cette décentralisation au niveau sectoriel existe en particulier parmi plusieurs pays regroupés dans le système coopératif de notre typologie et selon des formes diverses. Le financement de ces regroupements sectoriels est souvent assuré par le biais d'une cotisation sur les masses salariales des entreprises. À titre d'exemple, on retrouve au Danemark quinze fonds sectoriels couvrant environ la moitié de la main-d'œuvre et qui ont pour objet de financer les activités sectorielles conduisant à l'amélioration des qualifications de la maind'œuvre (détermination des besoins, élaboration des cours, détermination des qualifications par profession, etc.). Dans les Pays-Bas, les organisations patronales et les syndicats ont créé, sur des bases volontaires, soixante-dixhuit "Fonds sociaux industriels" qui sont financés par des cotisations sur les masses salariales (en moyenne $0,44 \%$ ) et qui ont aussi pour objet de soutenir des activités sectorielles reliées à la formation de la main-d'œuvre (Gasskov $1998: 37$ ).

Par ailleurs, de tels regroupements sectoriels ou intermédiaires (parfois régionaux) existent aussi dans des pays qui ne s'inscrivent pas forcément dans la même logique du système coopératif "à l'allemande ". Tel est le cas de la France, où il existe quelque quatre-vingt-dix organismes paritaires collecteurs agréés qui agissent comme des fonds de formation dont le financement provient en partie de l'État et en partie des entreprises (Gasskov 1998 : 33). Ce modèle français a inspiré certains comités sectoriels québécois dans les années $90^{18}$. Mentionnons aussi que même aux États-Unis, classés parmi les pays du modèle libéral reposant sur une démarche volontaire de l'entreprise, il existe des formes de regroupement sur des bases régionales ou sectorielles. Tel est le mandat particulier du National Skill Standards Board établi depuis 1994 qui vise à regrouper les différents acteurs du marché du travail et de l'éducation afin de conduire à la définition de normes professionnelles qui exerceraient alors un rôle directeur sur le contenu de la formation et des qualifications jugées nécessaires pour évoluer

18. Par exemple, le comité sectoriel de la plasturgie au Québec s'est inspiré de son équivalent français Plastifaf. 
sur le marché du travail. De portée plus locale, mentionnons aussi le cas des industriels du secteur de la production de vêtements de New York (regroupant 4600 fabriques ayant en moyenne 21 salariés) qui ont mis sur pied une association sectorielle gérée par un conseil tripartite ${ }^{19}$ visant particulièrement la formation de la main-d'œuvre du secteur et ce, pour toutes les catégories de main-d'œuvre. Cette association a conduit à la mise sur pied d'institutions de formation spécifiquement dédiées au secteur où l'on forme chaque année plus de 1000 ouvriers et cadres. On y retrouve aussi un service de placement de main-d'œuvre (BIT 1998: 120-121).

Cette tendance à la décentralisation se remarque ainsi dans plusieurs pays industrialisés et prend la forme de regroupements sectoriels et régionaux, selon les travaux récents du BIT. Elle correspond à une logique organisationnelle de mise en réseau d'entreprises ayant des besoins assez similaires en matière de formation, mais souvent incapables d'y répondre isolément les unes par rapport aux autres (BIT 1998:111-118). Il reste qu'une telle tendance à la décentralisation a aussi ses limites comme l'indique le BIT :

Mais la décentralisation excessive n'a pas que des avantages : elle peut compromettre la réalisation des objectifs nationaux en donnant aux acteurs locaux la possibilité de faire passer leur intérêt particulier au-dessus de l'intérêt national. La certification est un excellent exemple : elle doit être structurée à l'échelle nationale, faute de quoi ni les forces du marché ni les interventions de l'État ne seront efficaces. La décentralisation peut aussi entraîner des problèmes de coordination et des coûts transactionnels excessifs. Ce risque est particulièrement grand quand les besoins de formation et l'information sont à peu près homogènes partout et dans tous les secteurs. Si on abandonne la structure sectorielle pour décentraliser la formation à l'échelle locale, elle risque de moins bien répondre à la demande du marché national du travail et des diverses branches d'activité. Comment optimiser la décentralisation des systèmes publics de formation ? Dans les pays avancés, on observe que la décentralisation progresse sur des bases territoriales et sectorielles (BIT 1998 : 111).

Aussi, le BIT souligne qu'il faut une étroite imbrication des décisions des acteurs présents à tous les niveaux d'un système de formation, soit national, régional, sectoriel, professionnel et local, comme en témoigne le cas allemand. Il nous semble qu'il s'agit là de leçons intéressantes, tirées d'expériences internationales, qui devraient guider les analyses et les stratégies des acteurs au Québec en matière de décentralisation et d'articulation des niveaux dans le système de formation.

19. La Garment Industry Development Corporation a été créée en 1984 notamment pour répondre à des problèmes de formation et d'accès à une main-d'œuvre compétente. La direction est assurée par des représentants des employeurs, des syndicats et de l'administration de la ville de New York. 
La troisième tendance qui a influencé les orientations québécoises en matière de formation est celle de l'encadrement législatif qui a pris forme avec l'adoption de la Loi favorisant le développement de la formation de la main-d'œuvre en 1995. L'État québécois a alors choisi d'utiliser la loi comme un levier pour stimuler le développement de la formation au Québec, tout en laissant une grande marge de manœuvre à l'entreprise. Ce faisant, l'État québécois a clairement indiqué l'insuffisance d'une seule démarche volontaire des employeurs en regard du développement d'une culture de la formation. Cette idée d'une contribution obligatoire fixée par une loi circulait depuis au moins le début des années 80 suite au rapport de la Commission d'étude sur la formation des adultes. Le choix de l'État québécois de procéder à l'adoption d'une telle mesure coercitive s'inspirait du modèle français, bien que les modalités de distribution de l'effort obligatoire soient moins précises.

Certains ont soulevé le caractère trop peu contraignant de la loi quant à la distribution des dépenses de formation, d'autres ont discuté du seuil minimal trop faible, trop élevé ou trop uniforme. Cela nous amène à la quatrième tendance qui a influencé les orientations québécoises au cours des années 90, celle du respect de l'autonomie décisionnelle des acteurs de l'entreprise. Notre analyse est que l'État québécois, tout en prônant une intervention plus coercitive, a essentiellement voulu donner le message que les acteurs devaient s'approprier l'enjeu de la formation professionnelle continue tout en leur reconnaissant un rôle stratégique quant aux modalités et aux décisions les mieux adaptées au contexte de chacun des secteurs, de chacune des entreprises. À cet égard, ce n'est pas le seuil du $1 \%$ ou les modalités de sa distribution qui importaient vraiment pour l'État dans un premier temps. La loi était simplement un message aux acteurs que la formation était dorénavant un enjeu incontournable pour les acteurs de l'entreprise eux-mêmes et que l'État considérait que ces acteurs étaient les mieux placés pour décider des actions à prendre. En ce sens, la loi vise à influencer les comportements des acteurs et non pas à se substituer à leurs propres processus décisionnels internes (qu'il s'agisse des employeurs, de la maind'œuvre ou de leurs représentants). Ce respect de l'autonomie s'inscrivait dans la foulée de l'Énoncé de 1991 qui avait évoqué clairement les limites de l'approche bureaucratique. Autrement dit, la logique de ce respect de l'autonomie des acteurs est que l'État doit accepter avec une telle loi-cadre de ne pas tout prédéterminer et de ne pas chercher à tout contrôler quant aux actions qu'il vise à stimuler. L'État a d'ailleurs renvoyé la responsabilité de la définition de plusieurs modalités d'application de la loi du $1 \%$ aux partenaires présents au sein du conseil d'administration de la SQDM de l'époque.

Ce sont ces acteurs qui ont ainsi prévu l'importance de connaître la distribution des sommes consacrées à la formation en entreprise, ainsi que 
certaines modalités d'utilisation de ces sommes, en demandant aux employeurs de répondre sommairement de leurs décisions dans un court rapport annuel. L'État a pour sa part prévu que la loi donnerait lieu à un bilan cinq ans après son entrée en vigueur (soit autour de juin 2000). Ces décisions des partenaires et de l'État attestent à notre avis d'une révision possible des modalités de la loi et surtout, de la nécessaire réévaluation périodique de l'orientation de la politique publique. À cet égard, l'analyse qui en sera faite devrait reposer d'abord sur un partage d'informations communes entre tous les acteurs, dont des données quantitatives et qualitatives permettant de mesurer les résultats des dernières années. L'État joue à ce niveau un rôle essentiel dans la production d'informations qui assureront aux acteurs la possibilité de procéder à une évaluation rigoureuse. De plus, cette analyse devrait conduire à de nouveaux objectifs partagés par tous les acteurs. L'évaluation prochaine de la loi doit ainsi être perçue comme une étape dans un processus évolutif d'élaboration du système de formation, dont la loi du $1 \%$, rappelons-le, n'est qu'une des composantes.

\section{LES ACTEURS DU SYSTĖME DE RELATIONS INDUSTRIELLES AU QUÉBEC FACE À LA FORMATION : QUELLE ËVALUATION POUR L'ANNÉE 2000 ?}

Quelque dix ans après la publication de l'Énoncé de politique sur la main-d'œuvre, les acteurs québécois du système de relations industrielles sont invités à procéder à une première évaluation importante pour le début de l'an 2000, conformément aux dispositions de la loi du $1 \%$. Les modifications apportées au système de formation professionnelle au Québec sont encore récentes et les acteurs devraient se réserver de vouloir porter un jugement trop rapide sur un sujet aussi complexe que le système de formation, comme il faut éviter d'en analyser certaines composantes isolément des autres aspects introduits tout au long des années 90 . Cela n'enlève en rien toutefois à la légitimité, voire à la nécessité, de procéder à une évaluation continue des développements que connaît le système de formation professionnelle du Québec. Nous avons tenté de donner des points de repère pour contribuer à une telle évaluation. Concluons ici sur quelques éléments.

D'abord, il nous apparaît que le cadre analytique qui a sous-tendu les développements du système de formation au Québec dans la dernière décennie était partagé par plusieurs autres pays en réflexion quant au repositionnement de l'enjeu "formation " face au nouveau contexte économique des années 90 . La formation de la main-d'œuvre a été une préoccupation majeure dans les politiques publiques au cours de cette période et elle a occupé une place importante dans la littérature scientifique. En s'inscrivant dans cette trajectoire valorisant l'importance de la formation, 
les acteurs québécois n'ont certes pas erré. L'enjeu formation est-il maintenant devenu une chose du passé ? Si l'on se réfère aux développements en cours dans l'économie et sur le marché du travail, caractérisés par la place grandissante de ce que l'on nomme "l'économie du savoir ", il ne fait aucun doute que la formation demeurera un enjeu crucial du développement économique pour de nombreuses années à venir. La réflexion des acteurs québécois ne pourra pas faire abstraction de cette tendance.

Le système québécois de formation professionnelle est-il aujourd'hui davantage porteur d'une " culture commune de la formation " ? Rappelons qu'un des objectifs de l'Énoncé de 1991 était de rapprocher les acteurs de l'entreprise de l'enjeu formation et de transformer en quelque sorte les comportements. Pour ce faire, le Québec s'est inspiré de certaines pratiques étrangères visant une articulation plus étroite, mieux coordonnée des différents acteurs, en introduisant de nouveaux mécanismes intermédiaires et législatifs. Le gouvernement a cherché à organiser le marché de la formation et le partenariat plutôt que d'en attendre l'émergence par les seules pressions de l'environnement sur l'entreprise. À ce sujet, on peut souligner deux aspects en guise de résultats. D'une part, malgré certaines contradictions dans les décisions de l'État qui ont conduit à l'abolition de la structure centrale de partenariat qu'était la SQDM, il faut constater que l'émergence de plus d'une vingtaine de comités sectoriels de main-d'œuvre au cours des années 90 témoigne d'une volonté des acteurs de l'entreprise de s'engager dans de nouvelles formes de prise en charge de la formation. L'expérience est encore jeune pour la plupart de ces comités, mais certains d'entre eux ont fait la preuve de l'efficacité des structures intermédiaires pour contribuer au développement d'une culture de la formation dans les entreprises. L'émergence de ces comités est un premier indicateur de la responsabilisation des acteurs de l'entreprise envers l'enjeu formation. D'autre part, les quelques données disponibles quant au respect de l'exigence légale du $1 \%$ en formation révèle qu'au cours des premières années d'assujettissement à la loi, les entreprises rencontrent assez aisément l'objectif du $1 \%$. Il restera à voir si les entreprises seront à même de maintenir cet engagement sur plusieurs années et aussi, il faudra en savoir davantage sur la nature des dépenses effectuées en formation. L'effet de la loi pourra alors être mieux cerné quant au changement de comportement des employeurs envers la formation.

Soulignons aussi que l'analyse du système de formation professionnelle passe notamment par l'évaluation du rapport entre les fournisseurs de cette formation et ses destinataires. Plus rapide se fait l'adéquation entre les deux, plus dynamiques sont les échanges entre milieux de travail et milieux de l'éducation, plus efficace sera le marché de la formation, et cela pour le bien-être des fournisseurs, des jeunes, de la main-d'œuvre active et des entreprises. Pour y arriver, on ne peut considérer chacun de ces sous-systèmes 
du système de formation comme des entités cloisonnées. Il s'agit sans doute là d'un enjeu majeur de la société québécoise pour l'avenir de son système de formation et, en particulier, pour la contribution de son réseau public d'éducation à ce système. Or, une des solutions identifiées au début des années 90 , puis formellement inscrite dans la loi, était la mise sur pied d'un régime d'apprentissage au Québec. Jusqu'à présent, un tel régime n'a pas encore vu le jour. Les acteurs du marché du travail et du système d'éducation devraient inclure cet état de fait à leur réflexion prochaine sur la situation du système de formation.

Quant à la comparaison avec d'autres systèmes nationaux, l'exercice demeure limité pour évaluer la performance du système québécois. Ces systèmes sont faits d'imbrications institutionnelles qui imposent une grande prudence dans les comparaisons (Maurice, Selier et Silvestre 1982 ; Hollingsworth et Boyer 1997). Par ailleurs, la simple mesure des effets de la formation est déjà difficile à établir dans l'entreprise elle-même et, a fortiori, au niveau agrégé. Il est donc périlleux de vouloir produire des comparaisons de résultats que les données nationales ne permettent pas. Il s'agit là d'un enjeu international déjà reconnu récemment par les pays membres de l'Organisation de coopération et de développement économiques (OCDE 1997) qui visent à obtenir une meilleure comptabilité économique de la formation dans les entreprises et au niveau national, sur des bases qui permettraient les comparaisons. La prochaine décennie sera sans doute celle de l'établissement d'indicateurs internationaux permettant de mesurer certains résultats de la formation professionnelle. Dans ce contexte, les acteurs québécois du système de relations industrielles devraient établir des objectifs de résultats clairs à atteindre et se doter d'instruments de mesure et d'évaluation de leurs actions.

\section{BIBLIOGRAPHIE}

BÉLANGER, Yves. 1989. « L'État québécois et l'administration de la main-d'œuvre ». L'administration publique québécoise : évolutions sectorielles 1960-1985. Yves Bélanger et Laurent Lepage, dir. Sillery : Presses de l'Université du Québec, 129-172.

BERNIER, Colette et Michèle BilOdEAU. 1997. "Restructurations du travail, formation professionnelle et nouveaux partenariats ». La représentation des salariés dans le contexte du libre échange et de la déréglementation. Sélection de textes du XXXIII ${ }^{e}$ Congrès de l'ACRI. Rick Chaykowski, Paul-André Lapointe, Guylaine Vallée et Anil Verma, dir. Québec: ACRI/Département des relations industrielles, Université Laval, 181-194.

Bernstein, Aaron et Paul Magnusson. 1993. "How Much Good Will Training Do? ». Business Week, 22 février, 76-77. 
Betcherman, Gordon, Kathryn MCMulden et Katie Davidman. 1998. La formation et la nouvelle économie. Ottawa : Réseaux canadiens de recherche en politiques publiques.

BosCH, Gerhardt. 1993. "La formation professionnelle et la réorganisation des relations industrielles en Allemagne ". La formation professionnelle, perspectives internationales. Gilles Laflamme et al, dir. Québec : publication conjointe Département des relations industrielles, Université Laval et Genève : Département de la Formation, Bureau international du Travail, 235-268.

BRUNO, Sergio. 1998. "Le gène manquant : le facteur qualité ". Formation professionnelle. Numéro thématique Le financement de la formation professionnelle: approches politiques. Publication du CEDEFOP, 1-5.

BüCHTEMAnN, Christoph F. et Dana J. SOLOFF. 1998. "L'enseignement, la formation et l'économie ". Formation professionnelle. Numéro thématique Le financement de la formation professionnelle: approches politiques. Publication du CEDEFOP, 9-23.

BUREAU INTERNATIONAL DU TRAVAIL (BIT). 1998. Rapport sur l'emploi dans le monde 1998-99. Employabilité et mondialisation, le rôle crucial de la formation. Genève : BIT.

CHAREST, Jean. 1998a. "Les syndicats et la formation de la main-d'œuvre dans le secteur privé au Québec au cours des années 90 ". Le Marché du Travail, vol. $19, n^{\circ} 10,5-10$ et 73-76.

CHAREST, Jean. 1998b. "The Configuration of Sectoral Human Resource Initiatives in Quebec in the 1990s ". Forging Business-Labour Partnerships. Morley Gunderson et Andrew Sharpe, dir. Toronto : University of Toronto Press, 131157.

COMMISSION D'ÉTUDE SUR LA FORMATION DES ADULTES. 1982. Apprendre: une action volontaire et responsable. Québec: Direction de l'édition du ministère des Communications.

Commons, John Rogers. 1934. Institutional Economics. Its Place in Political Economy. New York : MacMillan Company.

COMmons, John Rogers. 1950. The Economics of Collective Action. Madison : The University of Wisconsin Press.

CONSEIL ÉCONOMIQUE DU CANADA. 1992. Les chemins de la compélence, Éducation et formation professionnelle au Canada. Ottawa : Approvisionnements et Services Canada.

DÉVELOPPEMENT DES RESSOURCES HUMAINES CANADA (DRHC). 1995.Réalisations choisies des conseils sectoriels. Ottawa : DRHC.

EMPLOI ET IMMIGRATION CANADA. 1989. Le nouveau mode d'emploi. Énoncé de principes. Ottawa : Approvisionnements et Services Canada.

EMPLOI-QUÉBEC. 1998. Guide général. Loi favorisant le développement de la formation de la main-d'œuvre. Québec : Ministère de l'Emploi et de la Solidarité.

EwER, Peter. 1998. "The Training Strategies of the Australian Union Movement ". A paper presented at the Conference of the Labour Education and Training Research Network, May 24-26, 1998. Toronto : Centre for Research on Work and Society (York Univeristy). 
FRASER, Doug. 1996. The Training Guarantee: Its Impact and Legacy 1990-1994. Summary volume. Canberra (Australia) : Department of Employment, Education, Training and Youth Affairs.

FRAZIS, Harley, Maury GITTLEMAN, Michael HORRIGAN et Mary JOYCE. 1998. "Results from the 1995 Survey of Employer-Provided Training ". Monthly Labor Review, juin, 3-13.

FREY, Bruno S. 1985. Economie politique moderne. Paris : Presses Universitaires de France.

GASSKOV, Vladimir. 1998. "Taxes, congés de formation et conventions collectives: comment inciter les entreprises et les individus à investir dans la formation ". Formation professionnelle. Numéro thématique Le financement de la formation professionnelle: approches politiques. Publication du CEDEFOP, 30-41.

HASHIMOTO, Masanori. 1994. "Employment-Based Training in Japanese Firms in Japan and in the United States : Experiences of Automobile Manufacturers ". Training and the Private Sector. International Comparisons. Lisa M. Lynch, dir. Chicago : University of Chicago Press, 109-148.

HODGSON, Geoffrey M. 1988. Economics and Institutions. A Manifesto for a Modern Institutional Economics. Philadelphia : University of Pennsylvania Press.

HODGSON, Geoffrey M. 1998. "The Approach of Institutional Economics ". Journal of Economic Literature, vol. XXXVI, mars 1998, 166-192.

HOLlingSWORTH, J. Rogers et Robert BOYER, dir. 1997. Contemporary Capitalism. The Embeddedness of Institutions. Cambridge : Cambridge University Press.

LOCKE, Richard M. et Wade JACOBY. 1997. "The Dilemmas of Diffusion: Institutional Transfer and the Remaking of Vocational Training Practices in Eastern Germany ". Negotiating the New Germany. Can Social Partnership Survive? Lowell Turner, dir. Ithaca : Cornell University, 33-68.

LYNCH, Lisa M. 1994. "Introduction ". Training and the Private Sector. International Comparisons. Lisa M. Lynch, dir. Chicago : University of Chicago Press, 1-24.

MAURICE, Marc. 1993. "L'arrimage entre l'entreprise et le système d'éducation en matière de formation professionnelle : le cas de la France, de l'Allemagne et du Japon ». La formation professionnelle, perspectives internationales. Gilles Laflamme et al., dir. Québec : publication conjointe Département des relations industrielles, Université Laval et Genève : Département de la Formation, Bureau international du Travail, 43-72.

MAURICE, Marc, François SElLIER et Jean-Jacques SILVESTRE. 1982. Politique d'éducation et organisation industrielle en France et en Allemagne. Paris : Presses Universitaires de France.

MINISTÈRE DE LA MAIN-D'(EUVRE, DE LA SÉCURITÉ DU REVENU ET DE LA FORMATION PROFESSIONNELLE (MMSRFP). 1989. Partenaires pour l'avenir : une stratégie de développement et de mise en valeur de la main-d'œuvre pour les années 1990. Position commune des provinces et territoires. Québec : Gouvernement du Québec MMSRFP.

MINISTÈRE DE LA MAIN-D' CEUVRE, DE LA SÉCURITÉ DU REVENU ET DE LA FORMATION PROFESSIONNELLE (MMSRFP). 1991. Partenaires pour un Québec compétent et compétitif. Québec : Gouvernement du Québec MMSRFP. 
NORTH, Douglas C. 1990. Institutions, Institutional Change and Economic Performance. Cambridge : Cambridge University Press.

OLSON, Mancur. 1978. Logique de l'action collective. Paris : Presses Universitaires de France.

ORGANISATION DE COOPÉRATION ET DE DÉVELOPPEMENT ÉCONOMIQUES (OCDE). 1997. Manuel pour élaborer de meilleures statistiques de la formation. Paris : OCDE.

PAQUET, Pierre. 1993. "L'évolution des politiques canadiennes et québécoises de formation des adultes depuis $1960 \%$. Enjeux actuels de la formation professionnelle. Pierre Dandurand, dir. Québec: Institut québécois de recherche sur la culture, 225-258.

PORTER, Michael E. 1990. The Competitive Advantage of Nations. New York : Free Press.

PORTER, Michael E. 1991. Le Canada à la croisée des chemins. Les nouvelles réalités concurrentielles. Une étude préparée pour le Conseil canadien des chefs d'entreprises et le Gouvernement du Canada. Ottawa : Conseil canadien des chefs d'entreprises et Ministre des approvisionnements et services.

POULIN SIMON, Lise et Judith CARROLL. 1991. "Historique des interventions du gouvernement du Québec dans le domaine de la main-d'œuvre ". Relations industrielles/Industrial Relations, vol. 46, $\mathrm{n}^{\circ} 4,766-801$.

RAPPORT DU CONSEIL CONSULTATIF SUR L'ADAPTATION (RAPPORT DEGRANDPRÉ). 1989. S'adapter pour gagner. Ottawa : Approvisionnements et Services Canada.

REICH, Robert B. 1992. The Work of Nations. New York : Vintage Books.

SABEL, Charles F. 1995. « Bootstrapping Reform : Rebuilding Firms, the Welfare State, and Unions ". Politics and Society, vol. 23, $\mathrm{n}^{\circ}$ 1, 5-48.

SACK, Jeffrey, Morley GunderSon, Donald SAVAGE et Jonathan EATON. 1993. "Comment combler le déficit canadien en matière de formation : initiatives publiques et privées dans les années quatre-vingt-dix ». La formation professionnelle, perspectives internationales. Gilles Laflamme et al., dir. Québec: publication conjointe Département des relations industrielles, Université Laval et Genève : Département de la Formation, Bureau international du Travail, 165-204.

SATO, Hiroki. 1993. "Japon : éducation permanente et formation en entreprise. Situation actuelle et principaux problèmes ". La formation professionnelle, perspectives internationales. Gilles Laflamme et al., dir. Québec : publication conjointe Département des relations industrielles, Université Laval et Genève : Département de la Formation, Bureau international du Travail, 111-128.

SHARPE, Andrew et Rodney HADDOW, dir. 1997. Social Partnerships for Training. Canada's Experiment with Labour Force Development Boards. Kingston: Caledon Institute of Social Policy and the School of Policy Studies, Queen's University.

SOSKICE, David. 1994. "Reconciling Markets and Institutions: The German Apprenticeship System ». Training and the Private Sector. International Comparisons. Lisa M. Lynch, dir. Chicago : The University of Chicago Press, 25-60. 
STEEDMAN, Hilary. 1993. « Grande-Bretagne : la formation professionnelle à l'école et en milieu de travail ». La formation professionnelle, perspectives internationales. Gilles Laflamme et al., dir. Québec : publication conjointe Département des relations industrielles, Université Laval et Genève : Département de la Formation, Bureau international du Travail, 73-90.

THuROW, Lester C. 1992. Head to Head: The Coming Economic Battle Among Japan, Europe and America. New York: William Morrow and Company.

VENNA, Yrjö. 1998. "Les interventions de l'État dans le domaine de la formation complémentaire à finalité professionnelle ". Formation professionnelle. Numéro thématique Le financement de la formation professionnelle : approches politiques. Publication du CEDEFOP, 50-63.

WEST, Anne, Hazel PENNELL et Ann EDGE. 1998. «L'introduction des principes de l'économie de marché dans le système de formation en Angleterre et au Pays de Galles ". Formation professionnelle. Numéro thématique Le financement de la formation professionnelle : approches politiques. Publication du CEDEFOP, $42-49$.

\section{SUMMARY}

Institutional Linkages and Future Directions for the Occupational Training System in Quebec

In the early 1990s, Quebec undertook to modernize its occupational training system through a new institutional linkage which entailed a significant and sustained commitment from the principal actors in industrial relations. In so doing, the government was responding to two factors : the strategy of its federal counterpart which, by the late 1980s, had already adopted strategic initiatives in the field of labour force development ; a more generalized trend towards making labour force training and development a key factor in economic development. Thus, the Quebec strategy was both a political and economic response to changes in the competitive environment.

Analysis of the state of the training system at the time largely informed this new policy direction. On the whole, Quebec's occupational training system was weak at all levels. There was no linkage both between public policies either within the government or between different levels of government; the institutional structure revealed unproductive tensions over the powers and cultures of the various actors ; the legislative framework for occupational training had not been modernized since 1969 ; employer and labour force behaviour (preferences, habits) often demonstrated little commitment to continuous manpower training; joint initiatives on the part of labour market actors were not very widespread, either at the level of the 
firm or beyond. Thus, as revealed by prevailing actor practices and institutional linkages, a "training culture" did not really exist in Quebec. Drawing on the observation of experiences abroad, the 1991 Policy Statement on Labour Force Development led to an important conclusion : a common training culture is not something that can be decreed or imported, it is an evolutionary social construction which requires active participation and commitment from all the actors.

This strategy of developing a "training culture" in Quebec attached great importance to the commitment of union and management actors within new regional, sectoral and national structures. As training was a societal issue, public policy required a form of partnership between union and management actors. Apart from this form of joint regulation, one of the characteristics of the new institutional linkage was the adoption of an act that forces employers to devote a minimum of one percent of their annual wage bill to training. This legislative provision was similar to the practice carried out in France since 1971 as well as in Australia between 1990 and 1994. The Quebec act is still very recent (gradually coming into effect since 1996), and there has not yet been any overall assessment of it. Such an assessment is, however, planned for the year 2000 and will undoubtedly be a crucial moment for the continuation of Quebec's strategy of training management.

Our analysis of the 1990s suggests four trends which have influenced the Quebec occupational training system. A first trend towards joint regulation, derived from neo-corporatism, has led to the establishment of new institutions linking the principal industrial relations actors to the management of public policy. However, the Quebec state has been variable in its support for this form of power sharing, and has, in recent years, reverted to traditional state control, giving rise to criticisms from industrial relations actors. The second trend is characterized by a decentralization which relates to an economic logic of bringing together actors in intermediary structures in order to respond to the issue of training, which is considered a public good. This decentralization, part of a common trend within different industrialized economies, is carried out through regional and sectoral committees for labour force development. The third trend is the legislative guidance which reflects the state's attempt to use the law as a lever to stimulate the development of training in Quebec, while leaving a wide margin of manœuvre for business actors. The fourth trend relates to the respect for business actors' autonomy in decision-making. Our analysis shows that, while supporting a more coercive intervention, the Quebec state basically sought to convey the message that actors should take charge of continuous manpower training, by recognizing their strategic role in the methods and decisions which are most appropriate to the context of each of the sectors and businesses. In this respect, what mattered most for the state was not the $1 \%$ threshold nor 
its method of distribution. The act was simply a way to convey the message to the actors that training was henceforth an issue that could not be ignored by business actors and the state considered that these actors were in the best position to make decisions about what actions should be taken. In this sense, the aim of the act is to influence the behaviour of actors and not to supersede their own internal decision-making processes (whether they are employers, workers or their representatives).

In our view, it is precisely the actors' autonomy or maturity in developing a "training culture" which will, in a way, be tested during the forthcoming public debates on training in Quebec. One thing is certain, the present economic environment does not in any way suggest that labour force training is no longer an economic development issue. It will be impossible for the actors to ignore this fact in future assessments of Quebec public policy on occupational training. 